\title{
The Neurospora crassa chs-2 gene encodes a non-essential chitin synthase
}

\author{
Adi Beth Din and Oded Yarden \\ Author for correspondence: Oded Yarden. Tel: +972 8 481298. Fax: + 9728 466794. e-mail: \\ YARDEN@HUJIAGRI.BITNET.AC.IL
}

Department of Plant Pathology and Microbiology, Faculty of Agriculture, The Hebrew University of Jerusalem, Rehovot 76100, Israel

\begin{abstract}
Chitin is a structural component of morphologically distinct structures assembled during various phases of growth and development in filamentous fungi. In Neurospora crassa, at least three different DNA fragments related to chitin synthase have been identified. In this study we cloned, sequenced and characterized the chitin synthase 2 structural gene (designated chs-2). The amino acid sequence deduced from the cloned chs-2 genomic DNA fragments is very similar to that of chitin synthase genes isolated from other fungi. Inactivation of the $\boldsymbol{N}$. crassa chs-2 gene by repeat-induced point (RIP) mutation produced progeny which under standard growth conditions were indistinguishable from the wild-type. However, a significant reduction in chitin synthase activity and increased sensitivity to the phosphatidylcholine biosynthesis inhibitor edifenphos are characteristic of the chs-2 ${ }^{\mathrm{RIP}}$ strain.
\end{abstract}

Keywords: Neurospora crassa, cell-wall biosynthesis, multiple chitin synthases, polyoxin D

\section{INTRODUCTION}

Chitin, a linear polymer of $N$-acetylglucosamine $(\mathrm{GlcN} A \mathrm{c})$ residues joined by $(1 \rightarrow 4)-\beta$ linkages, is one of the most abundant natural polymers. Its biosynthesis and deposition involve the sequential biotransformation of simple metabolites, polymerization of biosynthetic intermediates, and extrusion of the product outside the plasma membrane (Bartnicki-Garcia, 1989; Cabib, 1987; Peberdy, 1990; Ruiz-Herrera, 1992). Though abundant in arthropods, fungi and other eukaryotes, chitin is absent from plants and mammals (Muzzarelli et al., 1986). Thus, biosynthesis of this polymer is an attractive target in the development of anti-pest/anti-parasite drugs (Cohen, 1987; Gooday, 1990).

Chitin synthase activity is mainly associated with the membranous fractions of the fungal cell (Gooday, 1977) and has been partially purified from yeast and filamentous fungi (Bartnicki-Garcia et al., 1978; Gooday, 1977; Kang et al., 1984; Leal-Morales et al., 1988; Selitrennikoff, 1979). The genetics and molecular biology of chitin synthase in fungi were recently reviewed by Bulawa (1993). Several genes encoding chitin synthase polypeptides have been identified in the yeasts Saccharo-

Abbreviations: GICNAC, $\mathrm{N}$-acetylglucosamine; RFLP, restriction fragment length polymorphism; RIP, repeat-induced point mutation.

The EMBL accession number for the sequence reported in this paper is X77782. myces cerevisiae and Candida albicans, and their roles studied (Au-Young \& Robbins, 1990; Bulawa, 1992; Bulawa \& Osmond, 1990; Bulawa et al., 1986; Cabib et al., 1989; Chen-Wu et al., 1992; Duran et al., 1993; Shaw et al., 1991; Silverman et al., 1988; Sudoh et al., 1993). Chitinsynthase-like sequences have been deduced from cloned DNA fragments isolated from several filamentous fungi (Bowen et al., 1992), establishing the widespread occurrence of these genes in the fungal kingdom. However, the analysis of chitin synthase genes in filamentous fungi has not been as comprehensive as in yeast. To date, fulllength chitin synthase gene sequences from three filamentous species are available in databases [Aspergillus nidulans (N. Kojima, unpublished), Rhizopus oligosporus (T. Motoyama, unpublished), Neurospora crassa (Yarden \& Yanofsky, 1991)], but only the $N$. crassa chs-1 gene has been subjected to functional analysis (Yarden \& Yanofsky, 1991).

In this study we cloned, sequenced and characterized a second chitin synthase structural gene from $N$. crassa, and designated it $c h s-2$. We then used the repeat-induced point mutations (RIP) process (Selker, 1990) to inactivate the chromosomal chs-2 locus. Strains lacking a functional CHS2 have reduced chitin synthase activity (as measured in vitro), which is not accompanied by a reduction in cellwall chitin content. CHS2 activity is apparently not essential in vivo. 


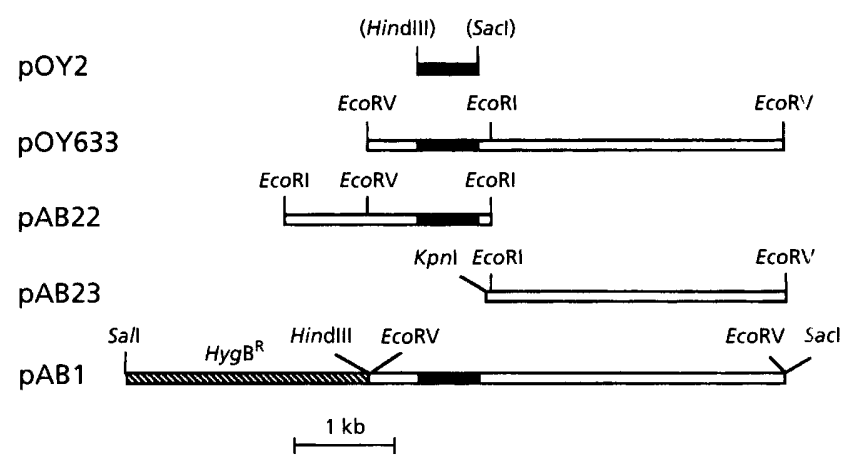

Fig. 1. Clones and constructs used in this study. pOY2 is a $N$. crassa genomic DNA product amplified by PCR and cloned into a Bluescript vector (the insert is from the NCCHS1 M13 clone provided by P.W. Robbins, Center for Cancer Research, Massachusetts Institute of Technology, USA). pOY633 is an EcoRV fragment obtained from a cosmid (pOY63) isolated from the Orbach/Sachs $N$. crassa genomic library, identified by hybridization with pOY2. pAB22 and pAB23 contain additional cloned fragments from the same cosmid. pAB1 is a $4 \mathrm{~kb}$ chs-2 fragment (identical to pOY633) cloned into pCSN43, a vector carrying the hygromycin phosphotransferase gene driven by the A. nidulans trpC promoter region. In all constructs shown, the Bluescript vector sequences have been omitted.

\section{METHODS}

Strains and media. Wild-type N. crassa strain 74-OR23-1A was used in all experiments. Procedures used in growth studies, crosses and other manipulations are described by Davis \& de Serres (1970). Cultures were maintained on $1.5 \%(\mathrm{w} / \mathrm{v})$ agar slants containing Vogel's minimal medium $N$ (Vogel, 1956). When appropriate, the medium was supplemented with hygromycin B (CalBiochem), polyoxin D or Hinosan (edifenphos; O-ethyl- $S, S$-diphenylphosphorothioate, ICI) at appropriate concentrations. The drugs were filter-sterilized and added to sterile media at approximately $50{ }^{\circ} \mathrm{C}$. DNA transformations of $N$. crassa were carried out as described by Orbach et al. (1986).

Isolation and analysis of nucleic acids from $\boldsymbol{N}$. crassa. Genomic DNA was isolated as follows: mycelia from cultures grown in $25 \mathrm{ml}$ Vogel's $\mathrm{N}$ medium were collected by filtration on Whatman No. 1 filter paper on a Büchner funnel. Samples were quick-frozen in liquid nitrogen and lyophilized. The dry samples were powdered by grinding and were suspended in an equal volume of lysis buffer $(50 \mathrm{mM}$ Tris $/ \mathrm{HCl}, \mathrm{pH} 8.0,50 \mathrm{mM}$ EDTA, $2 \%, \mathrm{w} / \mathrm{v}, \mathrm{SDS}, 1 \%, \mathrm{w} / \mathrm{v}, \beta$-mercaptoethanol) containing $25 \mu \mathrm{g}$ RNase $\mathrm{A} \mathrm{ml}^{-1}$. Following $1 \mathrm{~h}$ of incubation at $37^{\circ} \mathrm{C}, 100 \mu \mathrm{g}$ proteinase $\mathrm{K}$ (Boehringer Mannheim) $\mathrm{ml}^{-1}$ was added to the solution and incubation was continued for $1 \mathrm{~h}$ at $65^{\circ} \mathrm{C}$. Two phenol:chloroform $(1: 1, \mathrm{v} / \mathrm{v})$ extractions were followed by a single chloroform extraction, an isopropanol precipitation and a $75 \%(\mathrm{v} / \mathrm{v})$ ethanol wash. The DNA pellet was dried and dissolved in TE buffer $(10 \mathrm{mM}$ Tris/HCl, $\mathrm{pH} 8$; $1 \mathrm{mM}$ EDTA, pH 8*0).

The Orbach/Sachs $N$. crassa genomic library (Fungal Genetics Stock (entre) was screened as described by Benton \& Davis (1977). Southern analysis was carried out on nylon membranes (MagnaCharge, MSI) as described by Sambrook et al. (1989), as were all other DNA modification and cloning procedures. Bluescript SK - (Stratagene) was used for cloning and prep- aration of various constructs. pCSN43 (Staben et al., 1989) was used as a vector for $N$. crassa transformation. It carries the $h p h$ gene, encoding hygromycin phosphotransferase, which confers hygromycin resistance. This gene is driven by the $A$. nidulans $\operatorname{trpC}$ promoter region, and was used as a dominant selectable marker in the isolation of $N$. crassa transformants.

Mapping of $c h s-2$ was carried out by restriction fragment length polymorphism (RFLP) analysis of the 'small cross', according to the procedure of Metzenberg et al. (1985).

DNA sequencing and computer analysis. Double-stranded DNA was sequenced by the dideoxy chain-termination method (Sanger et al., 1977) with the aid of the Taq DyeDeoxy Terminator Cycle Sequencing kit and a 373A DNA Sequencer (Applied Biosystems). Purified DNA (Magic Miniprep; Promega) was used as template. Clones used for sequencing were generated by insertion of restriction fragments into the Bluescript vector. Oligonucleotide primers for sequencing portions of the gene were synthesized on an Applied Biosystems DNA synthesizer. Programs of The University of Wisconsin Genetics Group were used for analysis of nucleic acid and protein sequences (Devereux et al., 1984). The PAUP software package (Laboratory of Molecular Systematics, Smithsonian Institution) was used for maximal parsimony analysis among predicted chitin synthase polypeptides.

Fluorescent microscopy and chitin content determination. Samples were viewed with a Zeiss epifluorescence microscope. For chitin visualization, a drop of Calcofluor (Fluorescent Brightener 28, Sigma; $10 \mu \mathrm{g} \mathrm{ml}^{-1}$ ) was applied to fungal samples prespotted on a microscope slide. The filter combination used was $380-425 \mathrm{~nm}$ excitation, $430 \mathrm{~nm}$ dichroic mirror, and $450 \mathrm{~nm}$ barrier.

Chitin content was determined by a slight modification of the procedure described by Bulawa et al. (1986). Washed mycelium (2.5-5 mg dry weight) was lyophilized, suspended in $1 \mathrm{ml} \mathrm{6 \%}$ $(\mathrm{w} / \mathrm{v}) \mathrm{KOH}$ and heated for $90 \mathrm{~min}$ at $80^{\circ} \mathrm{C}$. Following the addition of $0 \cdot 1 \mathrm{ml}$ glacial acetic acid, the insoluble material was collected by centrifugation, washed twice with water, resuspended in $0.5 \mathrm{ml}$ sodium phosphate buffer $(\mathrm{pH} 6.3)$ and sonicated. A portion of $40-100 \mu$ l of a partially purified $(15 \%$ protein by weight) Streptomyces spp. chitinase suspension $\left(5 \mathrm{mg} \mathrm{ml} \mathrm{m}^{-1}\right)$ was added, and the tubes were incubated at $37^{\circ} \mathrm{C}$ for $2-20 \mathrm{~h}$. Following centrifugation, $450 \mu \mathrm{l}$ supernatant was treated with $40 \mu \mathrm{l} 50 \mathrm{mg} \beta$-glucuronidase type $\mathrm{H}-5$ (Sigma) $\mathrm{ml}^{-1}$ for $2 \mathrm{~h}$ at $37^{\circ} \mathrm{C}$. Portions $(0.1 \mathrm{ml})$ of each sample were removed and assayed for GlcNAc content (Reissig et al., 1955). In subsequent experiments, chitinases from Serratia marcescens (Sigma) or Aeromonas bydropbila (kindly provided by Y. Inbar and I. Chet, The Hebrew University of Jerusalem) were used.

Chitin synthase assays. Chitin synthase was assayed in fungal cell-free extracts. Extracts were prepared from 1.5-5-h-old germinating conidia, which were harvested by filtration (GF/C filter; Whatman). Following a water wash, the germlings were placed in a $2 \mathrm{ml}$ screw-cap tube (Sarstedt) containing $1.4 \mathrm{~g}$ $0.5 \mathrm{~mm}$-diameter zirconium beads (Biospec Products). The tube was then filled with cold $100 \mathrm{mM}$ HEPES, pH 7.8. The germlings were disrupted in a mini-bead beater (Biospec Products), using two $1 \mathrm{~min}$ pulses. The tubes were centrifuged for $10 \mathrm{~min}\left(4^{\circ} \mathrm{C}\right)$ and the supernatants collected. Protein concentration was determined by the method of Sedmak \& Grossbery (1977).

Chitin synthase activity was monitored in $25 \mu \mathrm{l}$ reaction mixtures containing $100 \mathrm{mM}$ HEPES, $\mathrm{pH} 7 \cdot 8,1 \mathrm{mM} \mathrm{MgCl}_{2}$, $32 \mathrm{mM}$ GlcNAc, $1 \mathrm{mM}$ UDP-GlcNAc and supplemented 
$\begin{array}{cccc}10 & 30 & 50 & 70 \\ \text { TGACCTGTCTGCGCCTCCACCTACTCTTGCATTGTCCCCAATTCCAGAGGGAACATCCCCGCGACGAGCGTCGCTTCGGGAGGCGCGCGAGAAGAACCAAAGAAGCAAGCAGCGTCTCAG }\end{array}$ $\begin{array}{ccccc}130 & 150 & 170 & 190 & 210\end{array}$ CAACATTACCGAAGAAAGGGACTCTACAAGATTCACTTACACCACCACCCTCGGTTCACTTTGGATCACCGAAAAGGCGAAACTCAGAAGCAGT ACGCTCACATATTATTCCTAMTACA

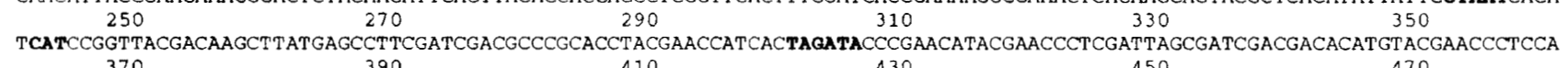
$\begin{array}{ccc}370 & 390 & 410 \\ \text { TCGACGAACGATCATCATACATGGATCCCCACGCATCCCACCACCCGACGGCGGATCTTACGTTTCTTCCTACATGGGCACCGAGAGCATGGTTTCGGGACACGGGAGACCATGGAGTC }\end{array}$

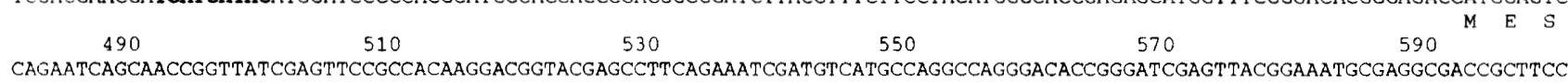

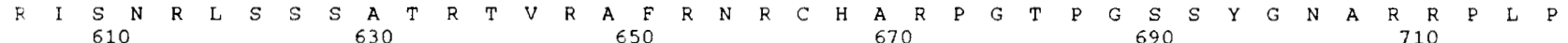
CTCGGCACCAGCGCCTTTACACTACAATAGCCCAAGTCGCGCAGCGAGTCATTATCCACGGTACCATGGAGGTTATGCGGACGACGTGACAGTTAGCATGGGACCGGACGACGATCGTAC

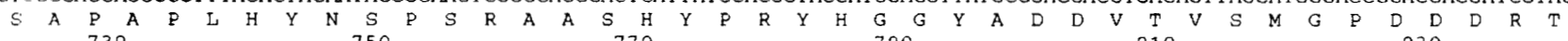

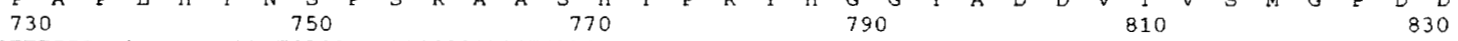
AGATATCTTTGGCCCCGAAACCGATCTCAGCGAAACGCGCCACCTCAACGACGCATACGGGTTTCGGTCATCCCAGATCACCCTCAGCGAAGATCCCCACGGCACCCACGCGCGTTCCCG

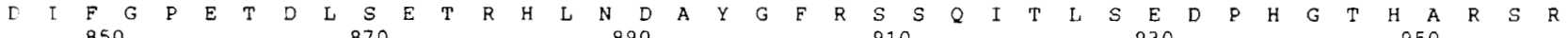
GT ACGACGACGAAGACGATGTGAGCACCACTTATTCCTCCAACACGGGCACCAGCGCTTCAGGTGTCGACAAGTTCGAGCATTACGGTCCCATTCCGGAGGAAGGCAAGCACGAGCGGCG

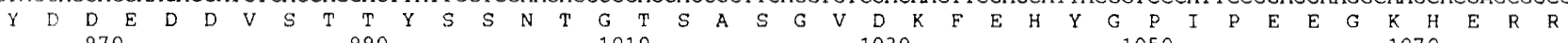

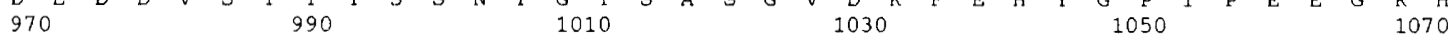

CGGCGTGCGACCACCACAGATGTCGAGGAAGGAAGTCCAGCTCATCAACGGCGAACTCGTTCTCGAGTGCAAGATTCCGACTATATTGTATTCGTTTT T'GCCCAGGAGAGACGAAGTGGA

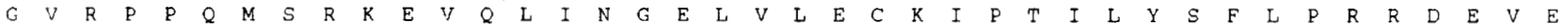

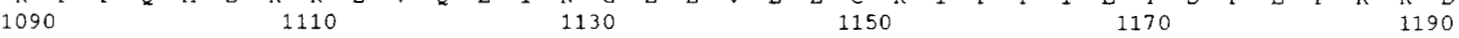

GT $m$ ACGCACATGCGGTACACAGCCGTCACTT GTGACCCTGATGACTTTGTTGCCAGGGGTTACAAGTTGCGCCAGAATATCGGTCGTACCGCCAGGGAGACGGAGCT GTTCATCTGCGT

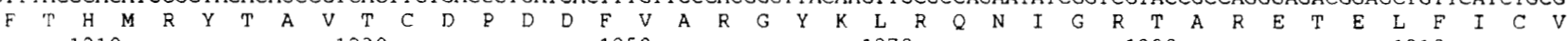

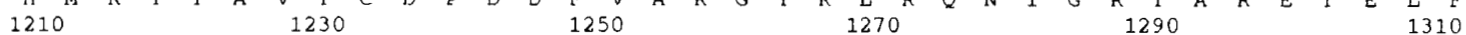

GACCATGTACAACGAGGACGAGTTCGGATTCACACGGACTATGCACGCAGTCATGAAGAACATTTCGCATTTTTGTTCCCGAAACAAGAGTAGGACGTGGGGAGCGGATGGGTGGCAGAA

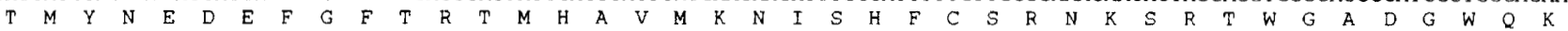

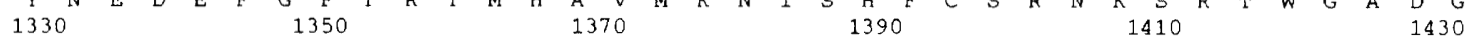

GATTGTGGTCTGTGTGGTTTCGGATGGACGAGAGATCATTCACCCCCGGACCTTGGACGCCCTCGCAGCCATGGGCGTTTACCAGCACGGTATCGCCAAGAACTTTGTCAACCAGAAGGC

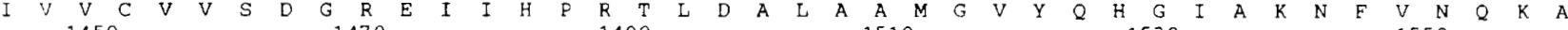
$1450 \quad 1470 \quad 1490 \quad 1510 \quad 1530 \quad 1550$

GGTGCAGGCCCACGTTTACGAGTACACGACACAAGTGTCTCTGGACAGCGACCTCATGTTCAAGGGCGCCGAGAAGGGCATCGTGCCCTGCCAGATGATT TTTTGCTTGAAGGAGAAGAA

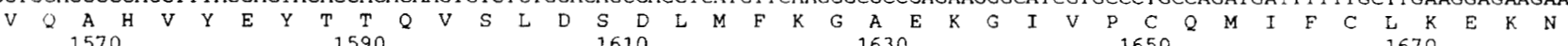

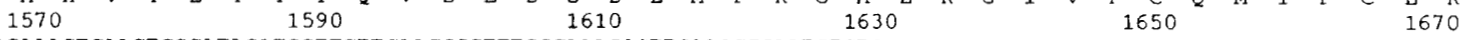

CCAAAAGAAACTCAACTCGCATAGATGGTTCTTCAACGCCTTTGGCAAAGCCTTGAACCCGAATGTGTGTATCCTCCT AGACGTCGGCACCCGCCCCGGCGGCACAAGTCTCTACCATCT

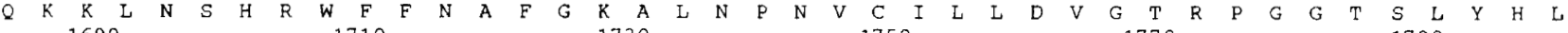
$1690 \quad 1710 \quad 1730 \quad 1750 \quad 1770 \quad 1790 \quad 0$

CTGGAAAGCCTTCGACACGGATTCCAACGTGGCGGGGGCCTGCGGGGAAATCAAAGCGATGAAGGGGCGGTTTGGCGGGAATTTGCTCAACCCTCTGGTGGCTAGTCAGAACTTTGAGTA

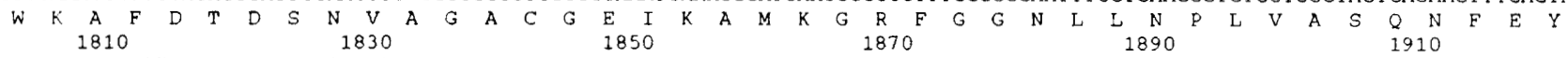

CAAGATGAGCAATATTCTGGACAAACCGTTGGAGTCGGTGTTTGGGTACATCACGGTGTTGCCGGGCGCCTTGTCGGCGTATCGGTACCATGCGCTGCAGAACGATGAGACGGGCCATGG

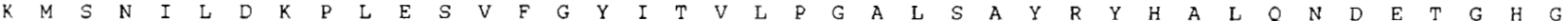

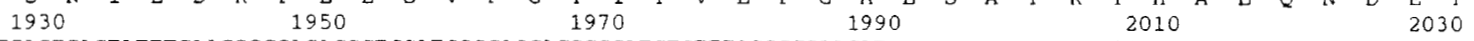

GCOGTTGAGTCAGTATTTCAAGGGCGAGACGCTCCATGGGCAGCACGCGGATGTGTTTACGGCGAACATGTACTTGGCCGAGGACCGAATTCTGTGTTGGGAGTTGGTGGCCAAGAGGGG

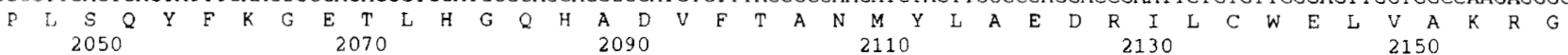

TGAGAGGTGGGTGTTGAAGTATGTGAAGGGGTGTACGGGTGAGACGGATGTGCCTGGTGAGTTGCCCCCCTTTTTTGCTGTGTGATAAGTTATACTAACGAGTTGTAGACACCGTCCCGG

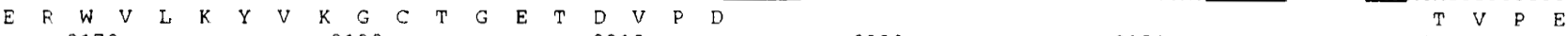
$\begin{array}{lcrrrr}2170 & 2190 & 2210 & 2230 & 2250\end{array}$

AATTCGTCTCGCAACGTCGTCGTTGGCTCAACGGTGCCTPCTTCGCCGCCGTCTACTCCCTCGTCCACTTTCGACAAATCTGGAAAACCGACCACACCTTTATGCGCAAAGCCCTTCTCC

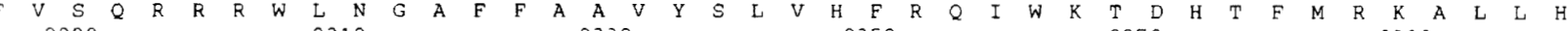
$2290 \quad 2310 \quad 2330 \quad 2350 \quad 2370 \quad 2390$

ACGTCGAATTCCTCTACCACCTCCTGCAACTCCTCTTCACCTACTTCTCCCTGGCCAACTTCTACCTCGCCTTCTACTMTATCGCCGGCGGTCTCGCCGATCCCCACGTCGACCCTTTTA

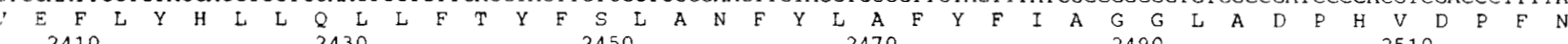

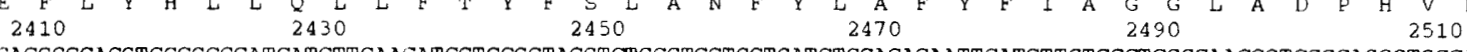

AC: CGGACGGCCACGTCGCGCGCATCATCTTCAACATCCTCCGCTACGTCTGCGTCCTGCTGATCTGCACACAATTCATCTTGTCCCTCGGCAACCGTCCGCAGGGTGCCAAAAGAATGT

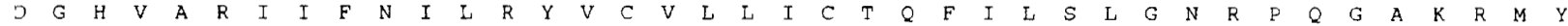
$2530 \quad 2550 \quad 2570 \quad 2590 \quad 2610 \quad 2630$

ATCTCGCATCCATGATCATCTACGCCGTCATCATGGTGTACACCACCTTCGCCACCATCTTCATCGTCGTGCGACAAATCCAACCCTCTCAAAAATCCGACGACAAGCCCGACCTCGAAC

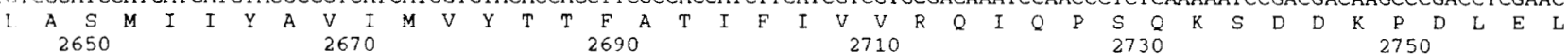

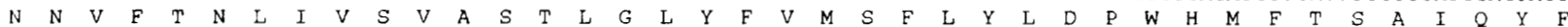

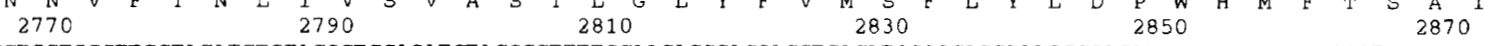

TTCTCCTGCTGCCTTCCTACATCTGTACGCTCCAGATCTACGCCTTTTGCAACACCCACGACGTCACATGGGGCACCAAAGGCGACAACGTGATGCGCACCGATCTCGGAGGCGCCATTG

$\begin{array}{llllllllllllllllllllllllllllllllllllllllllllll}V & L & L & P & S & Y & I & C & T & L & Q & I & Y & A & F & C & N & T & H & D & V & T & W & G & T & K & G & D & N & V & M & R & T & D & L & G & G & A & I & V\end{array}$ 29702990

TCAAGGGAAGCACCGTCGAACTGGAAATGCCTTCGGACCAACTCGACATCGACTCGGGATACGACGAATGTCTACGTAATCTCCGCGATCGCGTCATGGTGCCCTGCCGTTCCCTGTCCG

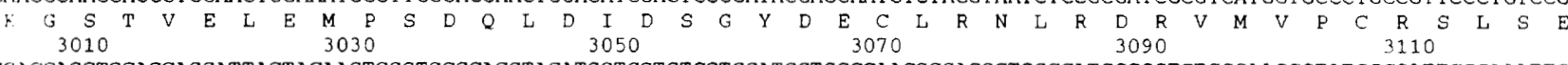

AGGACCAGCTGCAGCAGGATTACTACAAGTCGGTGCGCACGTACATGGTGGTGTCGTGGATGGTGGCCAACGCGACGCTGGCCATGGCGGTCTCGGAAGCGTATGGCGATTCGGAAATTG

$\begin{array}{lllllllllllllllllllllllllllllllllllllllll}Q & L & Q & Q & D & Y & Y & K & S & V & R & T & Y & M & V & V & S & W & M & V & A & N & A & T & L & A & M & A & V & S & E & A & Y & G & D & S & E & I & G & \end{array}$

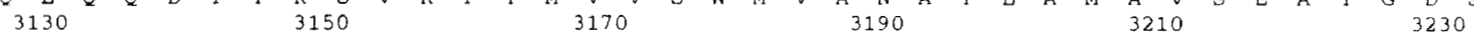

GGGATAATTTTTACTTGCGGTTTATCCTGTGGGCGGTGGCGGCCCTGGCGCTGTTTAGAGCGTTGGGGTCGACGACGTTTGCGGCGATTAATCTGGTGAGTGCTCTCGTGGAGGCCAGGG

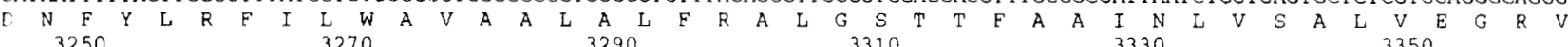

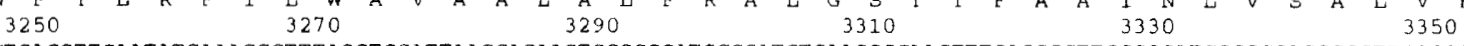

TCAGGCTGAGGTTGAATATGAAAGGGTTTAGGTGGATTAAGGAGAAGTGGGGGGATGCGGATGTGAAGGGCAAGTTTGAGGGGTTGGGGGATCGGGCGAGGGGGTTGGCGAAGCGGTGAT

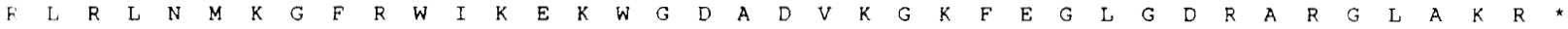

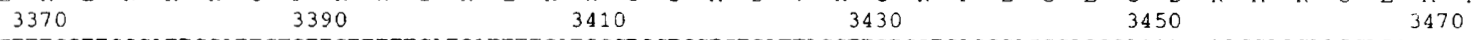
$\begin{array}{ccccc}\text { GTCTTTTTGGTTGGCATTCGATTCTGTTGTTTTTCATGATTTTCATGCCTGCTGCTGTGATTACGTTGTGGTCAGGCAGGCAGCGAGCAGCAAGCAGCAAGCAGCAAGCGAGCGAGCGA } & 3530 & 350 & 3570 & 350\end{array}$

CTTCGCTTCTTAAGGTTCGTTTTTTTCTPCTCTTTTTTGTTCCTCTTCTTCTTCTTTTCTTACGACGACATCTCACGACGTTACGAGATTCTTCT

Fig. 2. Complete nucleotide sequence of the $N$. crassa chs-2 gene and flanking regions and the predicted amino acid sequence of CHS2. The presumed CAAT, TATA, transcription start and polyadenylation signal sequences are shown in bold print. Conserved intron boundary and presumed lariat sequences are underlined. 
1

NCCHS2 AnCHSA ROCHS2 ROCHS1 NCCHS1 $\mathrm{AnCHSB}$

NCCHS2 AnCHSA ROCHS2 ROCHS1 NCCHS1 AnCHSB

NCCHS2 AnCHSA ROCHS2 ROCHS1 NeCHS1 AnCHSB

NCCHS2 AnCHSA ROCHS2 ROCHS NCCHS1 AnCHSB

$\mathrm{NCCHS2}$ AnCHSA ROCHS2 ROCHS1 NCCHS1 AnCHSB

NCCHS2 AnCHSA ROCHS2 ROCHSI $\mathrm{NCCHS}$ AnCHSB

NCCHS2 AnCHSA ROCHS2 ROCHS1 $\mathrm{NCCHS}$ AnCHSB

NCCHS2 AnCHSA ROCHS2 ROCHS 1 NCCHS1 AnCHSB

NCCHS? AnCHSA ROCHS2 ROCHS NCCHSI $\mathrm{AnCHSB}$

$\mathrm{NCCHS2}$ AnCHSA ROCHS2 ROCHS NCCHS2 AnCHSB

$\mathrm{NCCHS2}$ AnCHSA ROCHS2 RoCHS1 $\mathrm{NCCHS1}$ AnCHSE

NCCHS2 AnCHSA ROCHS2 ROCHS1 NCCHS1 AnCHSB $\ldots \ldots \ldots \ldots \ldots$
MPLRDRVLCH IMDCQNGRRA NRTVRFARTA

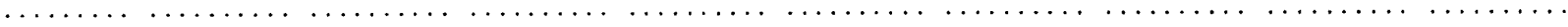

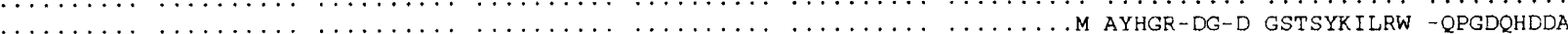

AASHYPRYH. ......... GGYADDVTVS MGPDD..DRT DIFGEETDLS ETRHLNDAYG FRSSQITLSE DPHG....T HARSR..Y YDD EDDVS ....T ---Y-E-ADI NGSPRPGTPS SR-GGSPRRP LP-APLFSKP GTTTQD-KID IGDGEE-PF- GGGRT-SSRH G-Q-SVQSF- SESTF... IA- -T-LEKVDLD .........MY PEGPKPEHDQ FPLQ-TQFSN QP-VHRSPFE -PYPEDQPHF DKQP-LTSPA YPPT-YPT-P P-PNFPGSPA VQQPYPPFNN NPSPVSPGVP .......... Y YP-NNSNNN- PM-SARLPSR PRY-GSEEYP MQQVSFNNNS PPPPPFPS-P NDT-FYDGND VQ-PL..LVN NGSTASSVTL

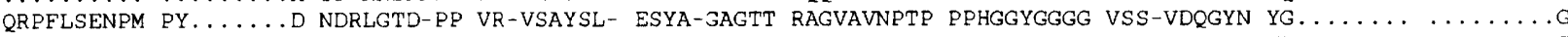
SHGLLSSQQS PFAGPFDDPH QQRGLTASPV QR-TSGYSL- ESYA-DAAYH $\ldots \ldots$-P-S ANQ-VYSGH. .SENPAAAFG VP...........

250

300

TYSSNTGTSA SGVDKFEHYG PIPEEGKHER RGVRPPQMSR KEVQLINGEL V.LECKIPTI LYSFLPRR. . ....DEVEF THMRYTAVTC DEDDFV.ARG

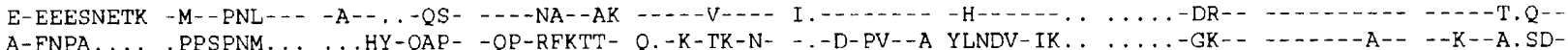

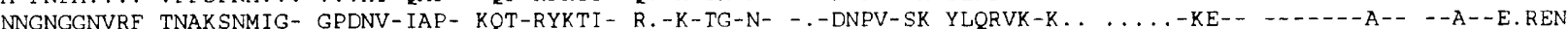
D-ATDPAYRM -AI-EDDSWL RRQQPNAAPT G-LKRYAT., RK-K-VQ-SV LS-DYPV-SA IRNAVQPKYR DE.EGNNE-- FK--.--A-- - -N--TLKNRVA..SPYAR -ETSST-AWR QRQAGARRGG N-L-RYAT. RK-K-VQ-SV LSVDYPV-SA IQNAIQAKYR NDLEGGSE-- -..---A-- - NE-TLHN301.350

YKLRQNIGRT ARETELFICV TMYNEDEFGF TRTMHAVMKN ISHFCSRNKS ..RTWGADGW QKIVVCVVSD GREIIHPRTL DALAAMGVYQ HGIAKNFVNQ -...-Q M-...-I - - PM, . L G-K -N--PAM. . Y N-H---L-AI -Y---KTLT A--L-G--Q- -RDIVNLKK- EFWNK-GPA- - - - - L-F- - IDPCDKD-- -V--TV-I-- D-VM-RD-DG 401 450

KAVQAHVYE. YTTQVSLDSD LMF.KGAEKG ...IVPCQMI FCLKEKNQKK LNSHRWFFNA FGKALNPNVC ILLDVGTRPG GTSLYHLWKA FDTDSNVAGA

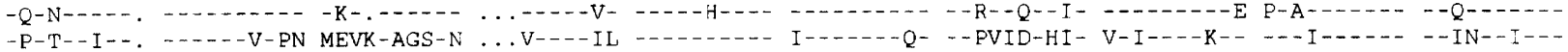

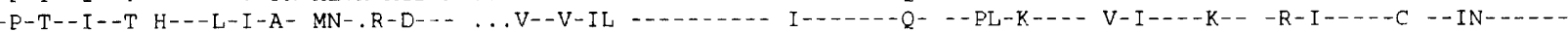

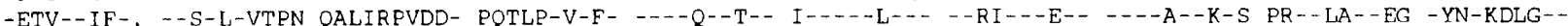

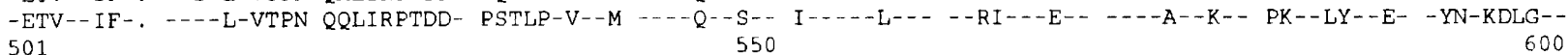
CGEIKAMKGR FGGNLLNPLV ASQNFEYKMS NILDKPLESV FGYITVLPGA LSAYRYHALQ NDETGHGPLS QYFKGETLHG Q......H ADVFTANMYL A- - - S- $-K$ NMLG- - R--A-T R-VA-... -A-.... --H--L-K G-KK-...-- -V-..--I- -......-A -- VS-...- F-.-FR-IM GR .....-E -.-H-DHTLS KLLGKKGIEG MNI-KK--F601 -H-L-K GWKK-...- -A--.-I AEDRILCWEL VAKRGERWVL KYVKGCTGET DVPDTVPEFV SQRRRWLNGA FFAAVYSLVH FRQIWKTDHT FMRKALLHVE FLYHLLQLLF TYFSLANFYL

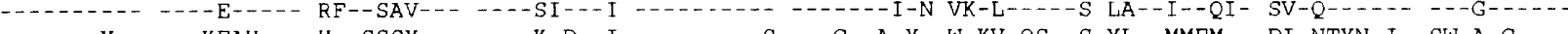

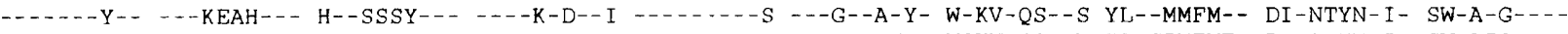
701 AFYFIAGGLA DPHVDPFNSD G........ VARIIFNILR YVCVLLICTQ FILSLGNRPQ GAKRMYLASM IIYAVIMVYT TFATIFIVVR QIQPSQKSD.

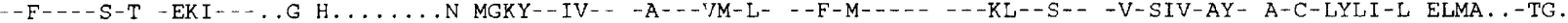
T-FILTKA-G HGVDGSTLT- P....PFSPD TGETLHTVFN - IYIV--VI - -MA-.... -S-IA-TS - VFF-IL--M M- -A-W-T-V GVKTVVETS. V--IMTTSIN ....... SVPN P.... PFAKN AGSVIHTV-N -LY-- -LII- I-IAM--.-- -Y-WA-FMI I VFF-IL-G-V L-C-AW-TIE GVKTAVETA. TTTV-MDLVG T-VTASSS-A EHHGWPFGDT -TPFFNAV-K -IYLAFVIL- - -A--.-K -S-WT-IT-F FVFSL-QS-I LVLSGYL-A-AFSVPLDQQL TTSV-MDLVG T-....SS-N -YTAFPFGKT ATP--NTLVK -IYLAFLLL- ---A-..--K -S-LS--- -F VAFGI-QL-V VVDALYL--- AFTGGAPM-F $801 \quad 850 \quad 900$ .. DKPDLE. . . . LGNNVFT NLIVSVASTL GLYFVMSFLY LDPWHMFTSA IQYFVLLPSY ICTLQIYAFC NTHDVTWGTK GDNVMRTDLG GA. IVKGS...

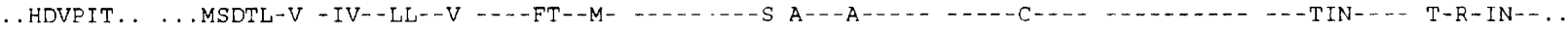

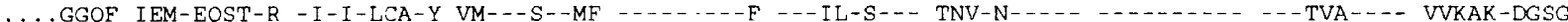

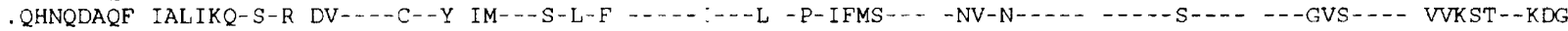

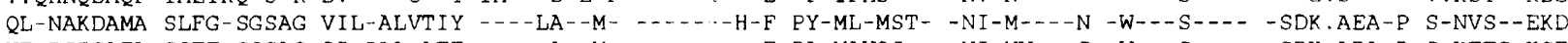

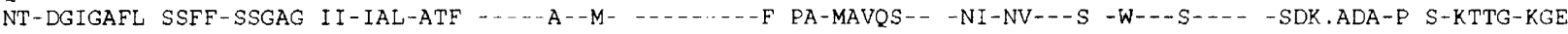
$901 \quad 950 \quad 1000$ ..TVELEMPS DQLDIDSGYD ECLRNLRDRV MVPCRSLSED QLQQD”YKSV RTYMVV. $\ldots \ldots \ldots$ SWMVAN ATLAMAVSEA $\ldots \ldots$. $\ldots \ldots \ldots \ldots$

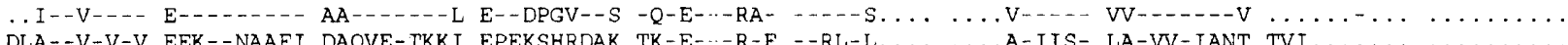
-RL-L.........A-IIS- LA-VN

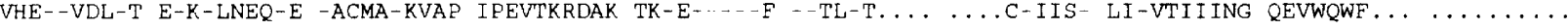
EAV--.-IEK P-E---QQFE ATV-RALAPY KEDETP..-P KDLE-(TSLS EPCLSCLGCF PTAS-RLLSP VITLTHLASG KPHPRELRGS SSSFCSLLVR EAVI-. - IDK P-A----QFE ATVKRALTPY VP-EEK...-E KSLD- $;---F-$-RL-TL... . . . . . LFS- GL--VCITSE GLDKFGFTVC FGCALSIIRS 1001 1050

.......GDS EIGDNFYLRF ILWAVAALAL FRALGSTTFA AINLV:ALVE GRVRLR.... . . LNMKGFRW IKEKWGDADV KGKFEGLGDR .......AR ........ VG SS-T-V--AI ---S--V--I I--I---AY- VLYLIOKL-- -KAKFQAGNI ASA-ASAAGS SLGTKSNVSY GS-GLNMT-- INETKWAIS-

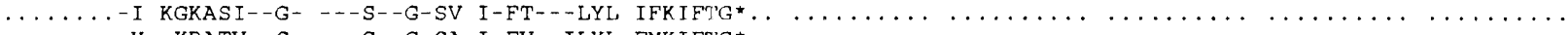

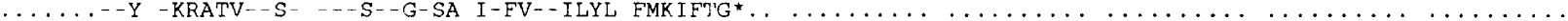
SLSSALLASV GSLAGR-YVL LCPSLSMANP KHVVVGACTL LTKRKEEEGR EKGYCFLAAA RRHGRVVP.$\ldots \ldots \ldots \ldots \ldots \ldots \ldots \ldots$

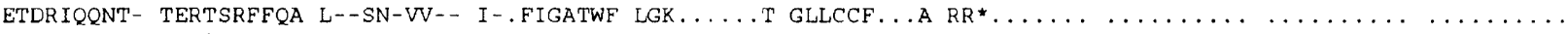
1101 1112

........

$\ldots \ldots \ldots$

.......

$\ldots \ldots \ldots$. 
with $1.5 \times 10^{-7} \mathrm{mM} \quad$ UDP $\left[1-{ }^{14} \mathrm{C}\right] \mathrm{GlcNAc} \quad\left[300 \mathrm{mCi} \mathrm{mmol}^{-1}\right.$ $\left(11 \cdot 1 \mathrm{GBq} \mathrm{mmol}^{-1}\right)$, Amersham, providing approximately $100(00$ d.p.m. per mixture]. Portions of the fungal cell-free extract used in each reaction mix contained $80 \mu \mathrm{g}$ protein. Cellfree extracts were trypsin activated; $2 \mu \mathrm{l} 0.2 \mathrm{mg}$ trypsin (EC 3.4.21.4; Sigma) $\mathrm{ml}^{-1}$ was added to the extracts, which were then incubated for $15 \mathrm{~min}$ at $30^{\circ} \mathrm{C}$. Soybean trypsin inhibitor was then added ( $2 \mu \mathrm{l} 0.3 \mathrm{mg} \mathrm{ml}^{-1}$ solution) to each reaction tube prior to the addition of the chitin synthase assay cock tail. Chitin synthase assay mixtures were incubated at $30{ }^{\circ} \mathrm{C}$ and reactions were terminated by the addition of $25 \mu \mathrm{l}$ glacial acetic acid. Reaction products were separated by paper chromatography using Whatman No. 1 paper and $4: 1(\mathrm{v} / \mathrm{v})$ ethanol: $1 \mathrm{M}$ acetic acid as solvent. Air-dried chromatograms were scanned and analysed with a Fuji Bioimaging BAS1000 analyser.

\section{RESULTS}

\section{Cloning of the $N$. crassa chs-2 gene}

A H lindIII-SacI fragment (approximately $500 \mathrm{bp}$ ) from a N. crassa PCR-amplified chitin-synthase-related sequence originally cloned in M13 (NCCHS1; Bowen et al., 1992) was cloned into a Bluescript SK - vector and designated pOY 2 .

With the $\sim 500$ bp pOY2 insert used as a probe, a genomic clone was identified and isolated from the Orbach/Sachs N. crassa genomic library. From the genomic cosmid clone (pOY63), a 4 kb EcoRV fragment, hybridizing to pOY2, was isolated, subcloned and designated pOY633 (Fig. 1). Two more overlapping fragments containing the entire $c h s-2$ gene were isolated from the same cosmid and subcloned (pAB22 and pAB23).

\section{Sequence of chs-2}

A $4 \mathrm{~kb}$ segment of pAB22 and pAB23 containing the chs2 gene was sequenced completely on both strands. The molecular organization of the gene, the complete nucleotide sequence and the predicted amino acid sequence of the CHS2 polypeptide are shown in Fig. 2. The chs-2 coding region was located by comparison of the predicted amino acid sequence with those of $N$. crassa chs1 and chitin synthase polypeptides predicted on the basis of genes described from two other filamentous fungi, $R$. oligosporus and A. nidulans (T. Motoyama, 1992 and N. Kojima, 1993, unpublished submissions to GenBank). The compiled $c b s-2$ reading frame shows a high bias for codons preferred by $N$. crassa (data not shown). The presumed $c h s-2$ start codon segment is GAGACCATGG. This segment is similar to that found in $N$. crassa chs-1 (Yarden \& Yanofsky, 1991) and aligns well with the sequence conserved in higher eukaryotes (Kozak, 1987). These sequences vary slightly from the $N$. crassa translation initiation consensus (Bruchez et al., 1993a).
Nucleotide sequences resembling putative CAAT and TATA boxes (CTAAT and TAGATA, respectively) were identified 241 and 172 nucleotides upstream of the tentative translation start site; also present is a TCATCATA element, which is highly conserved in $N$. crassa as a transcription start signal (Bruchez et al., 1993b; Fig. 2). The closest match to a polyadenylation signal present in eukaryotes (AATAAA; Proudfoot \& Brownlee, 1976) was an ATTA segment at nucleotides 3421-3424 (Fig. 2). One 57 bp intron was tentatively identified on the basis of consensus $5^{\prime}$ - and $3^{\prime}$ - splice junction sequences, characteristic of $N$. crassa introns (Bruchez et al., 1993a).

\section{Chromosomal localization of chs-2}

RFLP analyses were used to map chs-2. Of several restriction enzymes used ( $A p a \mathrm{I}, B a m \mathrm{HI}$, EcoRI, HindIII,

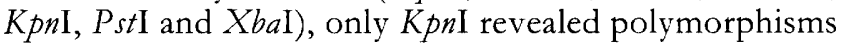
in the vicinity of $c b s-2$ in $N$. crassa of Oak Ridge and Mauriceville backgrounds (FGSC numbers 4411-4430). $K p n \mathrm{I}$ was used to digest DNA from progeny of the 'small cross' (Metzenberg et al., 1985). Subsequent probing with a hexamer-labelled HindIII-SacI insert of pOY2 located chs- 2 between nit-3 and 00031 on linkage group IV. The chs-2 polymorphism pattern was identical to that of $5 \mathrm{~S}$ rRNA gene Fsr-4 (data not shown). GCAG repeats as well as a TC-rich region very similar to sequences of $5 \mathrm{~S}$ RNA genes (Selker et al., 1981) were found downstream of the $3^{\prime}$ end of $c h s-2$ (Fig. 2). This, together with the fact that RFLP mapping showed $c h s-2$ to be linked to Fsr-4, raises the possibility that these two genes may be adjacent, even though the mapping procedure provides only relatively crude evidence with regard to physical distances.

\section{CHS2 polypeptide and amino acid homology}

The $c h s$-2 gene encodes a predicted 945-residue polypeptide with a calculated mass of $107 \mathrm{kDa}$ and a pI of 8.0. The predicted amino acid sequence of $N$. crassa CHS2 is $39 \%, 41 \%, 49 \%, 51 \%$ and $63 \%$ identical to those of $N$. crassa CHS1, A. nidulans CHSB, R. oligosporus CHS2 and CHS1 and $A$. nidulans CHSA, respectively. The most pronounced regions of predicted amino acid identity span amino acids $280-650$ and $840-890$ (Fig. 3). N. crassa CHS2 is also very similar to the $S$. cerevisiae and $C$. albicans zymogen-type chitin synthases (41-52\% identity with the predicted polypeptides). Based on the Bowen et al. (1992) nomenclature, the CHS2 polypeptide is a class I chitin synthase. The similarity in predicted amino acid sequence is also reflected in similar calculated pI values and molecular masses. Hydrophilicity analysis predicts a hydrophilic region at the amino terminus of the poly-

Fig. 3. Multiple sequence alignment of six predicted zymogen-type chitin synthase polypeptides. The comparison was of $N$. crassa CHS2 (NcCHS2) to N. crassa CHS1 (NcCHS1), two A. nidulans polypeptides (AnCHSA and AnCHSB) and two $R$. oligosporus polypeptides (RoCHS1 and RoCHS2). Periods (.) mark gaps; dashes (-) mark amino acid residues identical to the $N$. crassa CHS2 polypeptide. The $A$. nidulans and $R$. oligosporus sequences were predicted on the basis of unpublished nucleotide sequences available from GenBank (submitted by N. Kojima and T. Motoyama, respectively). 


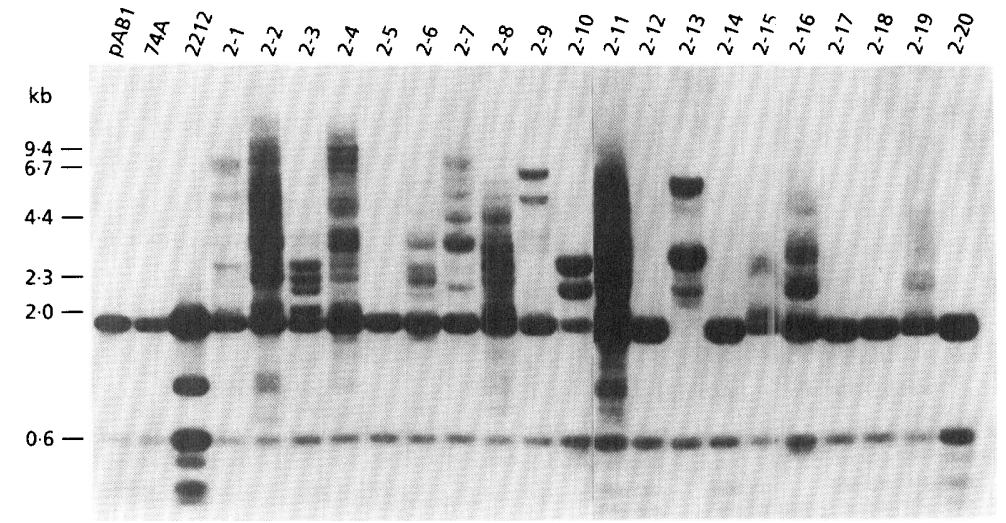

Fig. 4. Molecular analysis of chs-2 DNA in the wild-type strain, a pAB1-transformed strain, and progeny from a cross between a transformed parent and the wild-type. DNA samples from $p A B 1$, wild-type (74A), transformed parent (2212) and 20 progeny (2-1-2-20) from the cross were digested with Hpall, electrophoresed and blotted onto a nylon membrane. The blot was probed with the hexamer-labelled $4 \mathrm{~kb}$ EcoRV insert from pOY633. The strain $2-13$ is a chs- $2^{\mathrm{RIP}}$ progeny. peptide. The presence of several putative membranespanning domains near the carboxyl terminus is consistent with the membrane association of chitin synthase activity.

\section{chs-2 gene inactivation}

In an attempt to analyse the function of $c h s-2$ we altered the gene by the RIP process, which brings about heavy cytosine methylation and many premeiotic $\mathrm{GC} \rightarrow \mathrm{AT}$ base-pair transitions in duplicated DNA sequences (Selker, 1990). The $4 \mathrm{~kb}$ EcoRV-SacI fragment (flanked by the Bluescript vector HindIII-SacI sites) from pOY 633 was cloned into pCSN43 (a vector carrying a hygromycin $B$ resistance cassette). This clone ( $\mathrm{pAB} 1$ ) was transformed into a wild-type strain. Two transformants, both containing a single copy of pAB1, were each crossed with a wild-type strain of the opposite mating type. Ascospores from these crosses gave rise to what appeared to be colonies in which the growth rate and morphology were indistinguishable from the wild-type. Initially, DNA from 20 random progeny were analysed to determine if RIP had occurred. Southern analysis revealed a change in hybridization pattern of at least one of the progeny (Fig. 4). The change, consisting of the appearance of novel HpaII bands larger than the bands present in the parental strains, points to the inability of the methylation-sensitive restriction enzyme to cleave the original HpaII sites. Such changes are characteristic of RIP. Analysis of 20 additional random progeny provided evidence for at least two additional progeny in which $c h s-2$ had undergone RIP. The $c h s-2^{\mathrm{R} I P}$ progeny were further examined macroscopically and microscopically for possible morphological consequences of $c h s-2$ gene inactivation. No differences between these progeny and the wild-type were observed with respect to germination rate, hyphal elongation, conidiation or hyphal and conidial morphology. In addition, no apparent effect on protoperithecia formation or completion of the sexual phase of the life cycle was observed in the $c h s-2^{\mathrm{RIP}}$ strain. The $c h s-2^{\mathrm{RIP}}$ strain that was initially designated progeny 2-13 (Fig. 4) was stained with Calcofluor white $[\mathrm{a}(1 \rightarrow 4)-\beta]$ linkage polysaccharide dye) and analysed by fluorescent microscopy. No changes in fluorescence intensities of hyphae, crosswalls or conidial septa staining were observed when compared to the wild-type, indicating that the chitin-rich structures were not affected by $c h s-2$ alteration. The $2-13 c h s-2^{\mathrm{RIP}}$ strain was used in all subsequent analyses.

\section{Chitin synthesis in the chs-2 ${ }^{\mathrm{RIP}}$ strain}

An in vitro chitin synthase assay was carried out to determine whether $c h s-2$ alteration had influenced the rate of UDP-GlcNAc incorporation as measured in cell-free extracts. Following chromatographic separation of the reaction products, the intensity of insoluble radioactive product (concentrated at the chromatogram origin) was visualized and measured with the aid of a bioimager. The relative incorporation of UDP-GlcNAc into insoluble product was calculated to be significantly lower in the $c h s-2^{\mathrm{RIP}}$ extracts than that in the wild-type control $\left[8 \mathrm{nmol} \mathrm{h}^{-1} \text { ( } \mathrm{mg} \text { protein }\right)^{-1}$ and $31 \mathrm{nmol} \mathrm{h}^{-1}$ (mg protein $)^{-1}$, respectively]. This was evident in several experiments in which measurements were carried out at different time points.

On the basis of these results we analysed the chitin content of the $c h s-2^{\mathrm{RIP}}$ strain, in order to determine whether the reduction in enzymic activity (as determined above) had a measurable effect on cell-wall chitin content. Lyophilized mycelial powder samples of the wild-type and $c h s-2^{\mathrm{RIP}}$ strains were hydrolysed in alkali and digested by chitinase and $\beta$-glucuronidase. The two samples were assayed for GlcNAc (Reissig et al., 1955). The GlcNAc content of the $c h s-2^{\mathrm{RIP}}$ strain was calculated to be $1.93 \pm 0 \cdot 3 \%$ of the total dry weight, closely resembling the GlcNAc content calculated for the wild-type control $(2.06 \pm 0.2 \%)$ and in agreement with the mycelial glucosamine content reported by Mahadevan \& Tatum (1965) and Schmit et al. (1975). Similar results were obtained in several experiments in which different bacterial chitinases and various incubation periods were tested.

We examined the sensitivity of the $c h s-2^{\mathrm{RIP}}$ strain to competitive and non-competitive inhibitors of chitin synthase. When examined for colony growth, the $c h s-2^{\mathrm{RIP}}$ strain was not found to be more sensitive to polyoxin $\mathrm{D}$ than the wild-type $\left(\mathrm{EC}_{50}\right.$ of both strains was approximately $100 \mathrm{mM}$ ), and no significant changes in dose-response curves were observed (data not shown). This is in contrast to the increased sensitivity to a related drug, Nikkomycin Z, conferred by chs-1 gene inactivation 
(Yarden \& Yanofsky, 1991). We examined the sensitivity of the $c h s-2^{\mathrm{RIP}}$ strain to the fungicide edifenphos, an organophosphorus phosphatidylcholine biosynthesis inhibitor that was shown to be a non-competitive inhibitor of chitin synthase in several fungal species (Binks et al., 1993; Robson et al., 1990). When grown on solid medium amended with Hinosan ( $250 \mathrm{mM}$ active ingredient), hyphal proliferation was significantly delayed in the chs$2^{R{ }^{\prime}}$ strain compared to that of the wild-type. This was expressed in the area of the $c h s-2^{\mathbf{R} \mathbf{I P}}$ strain colony, which after several days reached only $10-15 \%$ of wild-type growth. Thus, even though no morphological abnormalities were observed under standard laboratory conditions, an altered $c h s$ - 2 gene may render the organism more sensitive to edifenphos, and perhaps also to other fungal growth inhibitors.

\section{DISCUSSION}

In this study, we present evidence for the presence of a non-essential chitin synthase gene in $N$. crassa. The gene, chs-2, is the second zymogen-type chitin synthase from a filamentous fungus to be structurally and functionally analysed.

We cloned, mapped and sequenced $c h s-2$. The predicted CHS2 polypeptide has a similar pI value and hydrophobicity pattern to that of other fungal (yeast and filamentous) chitin synthases that have been sequenced. When compared to other predicted chitin synthase polypeptides, chs-2 most resembles the chs $A$ gene of $A$. nidulans. Bowen et al. (1992) suggested a chitin synthase class subdivision and presented a possible prediction of phylogenetic relationship on the basis of the difference among 32 tentative chitin synthase gene products of 15 fungal species. They pointed out that analysis of partial sequences limits the value of the calculations and they

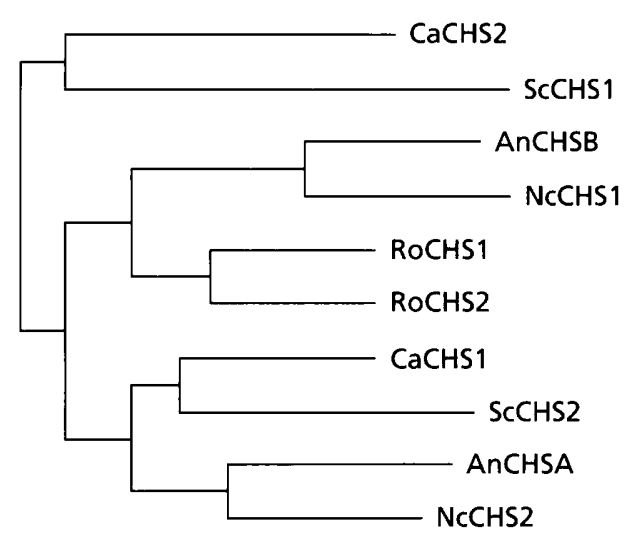

Fig. 5. Maximal parsimony dendrogram showing fungal $(\mathrm{A} n=A$. nidulans; $\mathrm{NC}=N$. crassa; $\mathrm{Ro}=R$. oligosporus; $\mathrm{Ca}=C$. albicans; $\mathrm{SC}=S$. (erevisiae) chitin synthase grouping. The tree was calculated by the program PAUP from deduced amino acid sequences. Branch length is relative to the number of amino acid substitutions. Neither rigorous calculation of evolutionary distances nor phylogenetic relationship can be inferred with confidence from this tree. Polypeptide designations are on the basis of EMBL entries. suggested extending the analysis to complete polypeptide sequences once they become available. We have carried out maximal parsimony similarity analysis of the ten available full chitin synthase polypeptides (Fig. 5). The obtained similarity tree is in close agreement with the dendrogram presented by Bowen et al. (1992), suggesting that partial sequence analysis (even of highly conserved regions of the polypeptide) may be sufficient for initial chitin synthase classification.

The RIP phenomenon was used to study the role of $c h s-2$ in vivo. In contrast to the consequence of $c h s-1$ inactivation (Yarden \& Yanofsky, 1991), progeny from crosses in which $c h s-2$ had undergone RIP did not exhibit any abnormal morphology. This was determined by both macroscopic (e.g. colony morphology, conidiation) and microscopic (hyphal morphology, Calcofluor-white staining) examination. Though a significant reduction (approximately $75 \%$ ) in in vitro chitin synthase activity was observed in the $c h s-2^{\mathrm{RIP}}$ strain, no striking decrease of cell-wall chitin accompanied the reduction in enzyme activity when compared to that measured in the wild-type. As in the $c h s-1^{\mathrm{RIP}}$ strain, crosswalls and conidial septa, where chitin is the major component (Hunsley \& Gooday, 1974), appeared to be normal in the $c h s-2^{\mathbf{R I P}}$ strain. The fact that the reduction in chitin synthase activity did not affect the cell-wall chitin content raises the possibility that compensation for loss of CHS2 activity may occur in vivo, presumably by another $N$. crassa chitin synthase gene product(s). Once additional $N$. crassa chitin synthase mutants (and multiple mutants) are obtained, the comprehensive analysis of this possibility can be carried out.

Even though there was no apparent morphological consequence of the RIP of the $c h s-2$ gene, it is possible that loss of $c h s-2$ may result in reduced fitness of the fungus. This is demonstrated by its increased sensitivity to the lipid-biosynthesis inhibitor edifenphos. On the other hand, in contrast to the $c h s-1^{\mathrm{RIP}}$ strain, which was shown to have increased sensitivity to nikkomycin Z (Yarden \& Yanofsky, 1991), no increase in sensitivity to polyoxin D (a structurally related competitive inhibitor of chitin synthase) was detected in the $c h s-2^{\mathrm{RIP}}$ strain. Thus, in contrast to the $S$. cerevisiae chitin synthases (Bulawa, 1993), it seems that not all $N$. crassa chitin synthases may be equally sensitive to the nikkomycin/polyoxin drug family. Alternatively, as the expression of different chitin synthase genes may be developmentally regulated, it is conceivable that our measurements, which were confined to mycelial growth on solid medium, have not exposed all sensitivities to this drug family. Further analysis of chitinsynthase-deficient mutants is being carried out to elucidate the differential sensitivity to chitin-biosynthesis inhibitors as well as to other antifungal compounds.

Even though RIP of $c h s-2$ was followed by a significant reduction in chitin synthase activity measured in vitro, the function of CHS2 in vivo has yet to be fully elucidated. So far, it is known not to be essential for normal growth and development under standard laboratory conditions. In $S$. cerevisiae, CHS1 was shown to encode for non-essential chitin synthase activity (Bulawa et al., 1986) and its involvement in repair functions was suggested (Cabib et 
al., 1989). It is possible that the $N$. crassa chs-2 gene may have a similar role.

We thank P. W. Robbins for the M13 NcCHS1 clone and Streptomyces sp. chitinase, and Y. Inbar and I. Chet for $A$. bydropbila chitinase. A. Beth Din was partially supported by a Gad Solovis Memorial Fellowship. This research was supported by the Wolfson Research Awards administered by the Israel Academy of Sciences and Humanities.

Au-Young, J. \& Robbins, P. W. (1990). Isolation of a chitin synthase gene (CHS1) from Candida albicans by expression in Saccharomyces cerevisiae. Mol Microbiol 4, 197-207.

Bartnicki-Garcia, S. (1989). The biochemical cytology of chitin and chitosan synthesis in fungi. In Chitin and Chitosan, pp. 23-36. Edited by G. Skjak-Braek, T. Anthonsen \& P. Sandford. London: Elsevier Applied Science.

Bartnicki-Garcia, S., Bracker, C. E., Reyes, E. \& Ruiz-Herrera, J. (1978). Isolation of chitosomes from taxonomically diverse fungi and synthesis of chitin microfibrils in vitro. Exp Mycol 2, 173-192.

Benton, W. \& Davis, R. (1977). Screening of lambda gt11 recombinant clones by hybridization to single plaques in situ. Science 196, 180-182.

Binks, P. R., Robson, G. D., Goosey, M. W. \& Trinci, A. P. J. (1993). Inhibition of phosphatidylcholine and chitin biosynthesis in Pyricularia oryzae, Botrytis fabae and Fusarium graminearum by edifenphos. J Gen Microbiol 139, 1371-1377.

Bowen, A. R., Chen-Wu, J. L., Momany, M., Young, R., Szaniszlo, P. J. \& Robbins, P. W. (1992). Classification of fungal chitin synthases. Proc Natl Acad Sci USA 89, 519-523.

Bruchez, J. J. P., Eberle, J. \& Russo, V. E. A. (1993a). Regulatory sequences involved in the translation of Neurospora crassa mRNA: Kozak sequences and stop codons. Fungal Genet New's/ 40, 85-88.

Bruchez, J. J. P., Eberle, J. \& Russo, V. E. A. (1993b). Regulatory sequences in the transcription of Neurospora crassa genes: CAAT box, TATA box, introns, poly(A) tail formation sequences. Fungal Genet Newsl 40, 89-96.

Bulawa, C. E. (1992). CSD2, CSD3, and CSD4, genes required for chitin synthesis in yeast: the CSD2 gene product is related to chitin synthases and to developmentally regulated proteins in Rbizobium and Xenopus. Mol Cell Biol 12, 1764-1776.

Bulawa, C. E. (1993). Genetics and molecular biology of chitin synthesis in fungi. Annu Rev Microbiol 47, 505-534.

Bulawa, C. E. \& Osmond, B. C. (1990). Chitin synthase I and synthase II are not required for chitin synthesis in vivo in Saccharomyces cerevisiae. Proc Natl Acad Si US A 87, 7424-7428.

Bulawa, C. E., Slater, M., Cabib, E., Au-Young, J., Sburlati, A., Adair, W. L. J. \& Robbins, P. W. (1986). The S. cerevisiae structural gene for chitin synthase is not required for chitin synthesis in vivo. Cell 46, 213-225.

Cabib, E. (1987). The synthesis and degradation of chitin. $A d v$ Enzymol 59, 59-101.

Cabib, E., Sburlati, A., Bowers, B. \& Silverman, S. J. (1989). Chitin synthase 1 , an auxiliary enzyme for chitin synthesis in Saccharomyces cervisiae. J Cell Biol 108, 1665-1672.

Chen-Wu, J. L., Zwicker, J., Bowen, A. R. \& Robbins, P. W. (1992). Expression of chitin synthase genes during yeast and hyphal growth phases of Candida albicans. Mol Microbiol 6, 497-502.
Cohen, E. (1987). Chitin biochemistry: synthesis and inhibition. Annu Rev Entomol 32, 71-93.

Davis, R. H. \& de Serres, F. J. (1970). Genetic and microbiological research techniques for Neurospora crassa. Methods Enzymol 17A, 79-143.

Devereux, J., Haeberli, P. \& Smithies, O. (1984). A comprehensive set of sequence analysis programs for the VAX. Nucleic Acids Res 12, 387-395.

Duran, A., Roncero, C., Santos, B. \& deCos, T. (1993). Biosynthesis of chitin in Saccbaromyces cerevisiae: effect of the fluorochrome calcofluor. In Chitin Enzymology, pp. 503-508. Edited by R. A. A. Muzzarelli. Ancona: European Chitin Society.

Gooday, G. W. (1977). Biosynthesis of the fungal cell wall mechanisms and implications. J Gen Microbiol 99, 1-11.

Gooday, G. W. (1990). Inhibition of chitin metabolism. In Biochemistry of Cell Walls and Membranes in Fungi, pp. 61-79. Edited by P. J. Kuhn, A. P. J. Trinci, M. J. Jung, M. W. Goosey \& L. G. Coppings. Berlin: Springer-Verlag.

Hunsley, D. \& Gooday, G. W. (1974). The structure and development of septa in Neurospora crassa. Protoplasma 82, 125-146.

Kang, M. S., Elango, N., Mattia, E., Au-Young, J., Robbins, P. W. \& Cabib, E. (1984). Isolation of chitin synthetase from Saccharomyces cerevisiae: purification of an enzyme by entrapment in the reaction product. J Biol Chem 259, 14966-14972.

Kozak, M. (1987). An analysis of 5'-noncoding sequences from 699 vertebrate messenger RNAs. Nucleic Acids Res 15, 8125-8148.

Leal-Morales, C. A., Bracker, C. E. \& Bartnicki-Garcia, S. (1988). Localization of chitin synthetase in cell-free homogenates of Saccharomyces cerevisiae: chitosomes and plasma membrane. Prov Natl Acad Sci USA 85, 8516-8520.

Mahadevan, P. R. \& Tatum, E. L. (1965). Relationship of the major constituents of the Neurospora crassa cell wall to wild-type and colonial morphology. J Bacteriol 90, 1073-1081.

Metzenberg, R. L., Stevens, J. N., Selker, E. U. \& MorzyckaWroblewska, E. (1985). Identification and chromosomal distribution of $5 \mathrm{~S}$ rRNA genes in Neurospora crassa. Proc Natl Acad $S_{c i}$ US A 82, 2067-2071.

Muzzarelli, R., Jeuniaux, C. \& Gooday, G. W. (1986). Chitin in Nature and Tecbnology. New York: Plenum Press.

Orbach, M. J., Porro, E. B. \& Yanofsky, C. (1986). Cloning and characterization of the gene for $\beta$-tubulin from a benomyl-resistant mutant of Neurospora crassa and its use as a dominant selectable marker. Mol Cell Biol 6, 2452-2461.

Peberdy, J. F. (1990). Fungal cell walls - a review. In Biocbemistry of Cell Walls and Membranes in Fungi, pp. 5-30. Edited by P. J. Kuhn, A. P. J. Trinci, M. J. Jung, M. W. Goosey \& L. G. Coppings. Berlin: Springer-Verlag.

Proudfoot, N. J. \& Brownlee, G. G. (1976). 3' non-coding region sequences in eukaryotic mRNA processing intermediates. Nature 263, 211-214.

Reissig, J. L., Stringer, J. L. \& Leloir, L. F. (1955). A modified colorimetric method for the estimation of $N$-acetylamino sugars. $J$ Biol Chem 217, 959-966.

Robson, G. D., Wiebe, M., Kuhn, P. J. \& Trinci, A. P. J. (1990). Inhibitors of phospholipid biosynthesis. In Biochemistry of Cell Walls and Membranes in Fungi, pp. 261-281. Edited by P. J. Kuhn, A. P. J. Trinci, M. J. Jung, M. W. Goosey \& L. G. Coppings. Berlin: Springer-Verlag.

Ruiz-Herrera, J. (1992). Fungal Cell Wall: Structure, Synthesis and Assembly. Boca Raton: CRC Press.

Sambrook, J., Fritsch, E. F. \& Maniatis, T. (1989). Molecular Cloning: 
A Lahnratory Manual. Cold Spring Harbor, New York : Cold Spring Harbur Laboratory Press.

Sanger, F., Nicklen, S. \& Coulson, A. R. (1977). DNA sequencing with chain-terminating inhibitors. Proc Natl Acad Sci USA 74, 54635467.

Schmit, J. C., Edson, C. M. \& Brody, S. (1975). Changes in glucosamine and galactosamine levels during conidial germination in Niturospora crassa. J Bacteriol 122, 1062-1070.

Sedmak, J. J. \& Grossbery, S. E. (1977). A rapid, sensitive and versatile assay for protein using Coomassie brilliant blue (G-250). Anal Biochem 79, 544-552.

Selitrennikoff, C. P. (1979). Chitin synthase activity from the slime variant of Neurospora crassa. Biochem Biophys Acta 571, 224-232.

Selker, E. U. (1990). Premeiotic instability of repeated sequences in Neuruspora crassa. Annu Rev Genet 24, 579-613.

Selker, E. U., Yanofsky, C., Driftmier, K., Metzenberg, R. L., Alzner-De-Weerd, B. \& RajBhandary, U. L. (1981). Dispersed 5S RN $\backslash$ genes in N. crassa: structure, expression and evolution. Cell 24, $819-828$.

Shaw, J. A., Mol, P. C., Bowers, B., Silverman, S. J., Valdivieso, M. H., Duran, A. \& Cabib, E. (1991). The function of chitin synthases 2 and 3 in the Saccharomyces cerevisiae cell cycle. J Cell Biol 114, 111-123.

Silverman, S. J., Sburlati, A., Slater, M. L. \& Cabib, E. (1988). Chitin synthase 2 is essential for septum formation and cell division in Saccharomyces cerevisiae. Proc Natl Acad Sci US A 85, 4735-4739.

Staben, C., Jensen, B., Singer, M., Pollock, J., Schectman, M., Kinsey, J. \& Selker, E. (1989). Use of a bacterial hygromycin B resistance gene as a dominant selectable marker in Neurospora crassa transformation. Fungal Genet News/ 36, 79-81.

Sudoh, M., Nagahashi, S., Doi, M., Ohta, A., Takagi, M. \& Arisawa, M. (1993). Cloning of the chitin synthase 3 gene from Candida albicans and its expression during yeast-hyphal transition. Mol \& Gen Genet 241, 351-358.

Vogel, H. J. (1956). A convenient growth medium for Neurospora crassa (medium N). Microb Genet Bull 13, 42-43.

Yarden, O. \& Yanofsky, C. (1991). Chitin synthase 1 plays a major role in cell wall biogenesis of Neurospora crassa. Genes \& Dev 5, $2420-2430$.

Received 7 March 1994; revised 19 May 1994; accepted 25 May 1994. 